\title{
Multiple solutions for some symmetric supercritical problems*
}

\author{
A.M. Candela, G. Palmieri, A. Salvatore \\ Dipartimento di Matematica \\ Università degli Studi di Bari Aldo Moro \\ Via E. Orabona 4, 70125 Bari, Italy \\ annamaria.candela@uniba.it \\ giuliana.palmieri.uniba@gmail.com \\ addolorata.salvatore@uniba.it
}

\begin{abstract}
The aim of this paper is investigating the existence of one or more critical points of a family of functionals which generalizes the model problem

$$
\bar{J}(u)=\frac{1}{p} \int_{\Omega} \bar{A}(x, u)|\nabla u|^{p} d x-\int_{\Omega} G(x, u) d x
$$

in the Banach space $X=W_{0}^{1, p}(\Omega) \cap L^{\infty}(\Omega)$, where $\Omega \subset \mathbb{R}^{N}$ is an open bounded domain, $1<p<N$ and the real terms $\bar{A}(x, t)$ and $G(x, t)$ are $C^{1}$ Carathéodory functions on $\Omega \times \mathbb{R}$.

We prove that, even if the coefficient $\bar{A}(x, t)$ makes the variational approach more difficult, if it satisfies "good" growth assumptions then at least one critical point exists also when the nonlinear term $G(x, t)$ has a suitable supercritical growth. Moreover, if the functional is even, it has infinitely many critical levels.

The proof, which exploits the interaction between two different norms on $X$, is based on a weak version of the Cerami-Palais-Smale condition and a suitable intersection lemma which allow us to use a Mountain Pass Theorem.
\end{abstract}

2000 Mathematics Subject Classification. 35J92, 35J20, 35J60, 58E05.

Key words. Quasilinear elliptic equation, weak Cerami-Palais-Smale condition, Ambrosetti-Rabinowitz condition, supercritical growth.

\section{Introduction}

Here, we look for critical points of the nonlinear functional

$$
\mathcal{J}(u)=\int_{\Omega} A(x, u, \nabla u) d x-\int_{\Omega} G(x, u) d x, \quad u \in \mathcal{D} \subset W_{0}^{1, p}(\Omega),
$$

${ }^{*}$ The research that led to the present paper was partially supported by Fondi di Ricerca di Ateneo "Metodi variazionali e topologici nello studio di fenomeni non lineari" and Research Funds INdAM - GNAMPA Project 2017 "Metodi variazionali per fenomeni non-locali" 
which generalizes the model problem

$$
\bar{J}(u)=\frac{1}{p} \int_{\Omega} \bar{A}(x, u)|\nabla u|^{p} d x-\int_{\Omega} G(x, u) d x, \quad u \in \mathcal{D} \subset W_{0}^{1, p}(\Omega),
$$

where $\Omega$ is an open bounded domain in $\mathbb{R}^{N}, 1<p<N, A: \Omega \times \mathbb{R} \times \mathbb{R}^{N} \rightarrow \mathbb{R}$, respectively $\bar{A}: \Omega \times \mathbb{R} \rightarrow \mathbb{R}$, and $G: \Omega \times \mathbb{R} \rightarrow \mathbb{R}$ are given functions.

We note that, even in the simplest case $A(x, u, \nabla u)=\frac{1}{p} \bar{A}(x, t)|\nabla u|^{p}$ and $G(x, t) \equiv 0$, with $\bar{A}(x, t)$ smooth, bounded away from zero but $\frac{\partial \bar{A}}{\partial t}(x, t) \not \equiv 0$, the functional $\bar{J}$ is defined in $W_{0}^{1, p}(\Omega)$ but is Gâteaux differentiable only along directions of $W_{0}^{1, p}(\Omega) \cap L^{\infty}(\Omega)$.

In the past, such a problem has been overcome by introducing suitable definitions of critical point for $\mathcal{J}$ and related existence results have been stated (see, e.g., [2, 3, 11, 15]). Here, as in [7], suitable assumptions assure that the functional $\mathcal{J}$ is $C^{1}$ in $X=W_{0}^{1, p}(\Omega) \cap L^{\infty}(\Omega)$ (see Proposition 3.2) and its Euler-Lagrange equation is

$$
\begin{cases}-\operatorname{div}(a(x, u, \nabla u))+A_{t}(x, u, \nabla u)=g(x, u) & \text { in } \Omega, \\ u=0 & \text { on } \partial \Omega,\end{cases}
$$

where

$$
A_{t}(x, t, \xi)=\frac{\partial A}{\partial t}(x, t, \xi), a(x, t, \xi)=\left(\frac{\partial A}{\partial \xi_{1}}(x, t, \xi), \ldots, \frac{\partial A}{\partial \xi_{N}}(x, t, \xi)\right), G(x, t)=\int_{0}^{t} g(x, s) d s .
$$

We note that, from a physical point of view, problem (1.2) is interesting for its applications. For example, if $\Omega=\mathbb{R}^{N}$ and $A(x, u, \nabla u)=\left(1+|u|^{2}\right)|\nabla u|^{2}$, model equations of (1.2) appear in Mathematical Physics and describe several physical phenomena in the theory of superfluid film and in dissipative quantum mechanics (for more details, see [14 and references therein).

In order to find solutions of (1.2), i.e. critical points of $\mathcal{J}$ in $X$, we cannot apply directly existence and multiplicity results similar to the classical Ambrosetti-Rabinowitz theorems (see [1, 5]). Indeed, our functional $\mathcal{J}$ does not satisfy the Palais-Smale condition in $X$ as it has PalaisSmale sequences which converge in $W_{0}^{1, p}(\Omega)$ but are unbounded in $L^{\infty}(\Omega)$ (see, e.g., 9, Example 4.3]). Hence, we have to weaken the definition of Palais-Smale condition (see Definition 2.1) and use it for stating a generalized version of the Mountain Pass Theorems (see Theorems 2.3 and 2.4).

In [7] the existence of critical points of the functional $\mathcal{J}$, i.e. solutions of (1.2), has been already proved if $p>1, A(x, t, \xi)$ satisfies suitable assumptions and $G(x, t)$ has a $p$-superlinear growth which has to be subcritical if $p<N$. Anyway, even if the dependence from $t$ of the principal part $A(x, t, \xi)$ makes the variational approach more difficult, it can allow the nonlinear term $G(x, t)$ to be supercritical. In fact, the aim of this paper is to extend the main statements in [7] to a function $G(x, t)$ with critical or supercritical growth if $1<p<N$ : roughly speaking, we prove that the more $A(x, t, \xi)$ is unbounded and grows with respect to $t$, the more $G(x, t)$ can have a supercritical growth.

Since our main theorems need a list of hypotheses, we give their complete statements in Section 4 (see Theorems 4.1 and 4.3), anyway, here, in order to highlight how our approach improves previous results, we consider the particular setting

$$
A(x, t, \xi)=\frac{1}{p}\left(A_{1}(x)+A_{2}(x)|t|^{p s}\right)|\xi|^{p} \quad \text { and } \quad g(x, t)=|t|^{\mu-2} t
$$


with $1<p<N, s \geq 0, \mu \geq 1$, so that problem (1.2) reduces to

$$
\begin{cases}-\operatorname{div}\left(\left(A_{1}(x)+A_{2}(x)|u|^{p s}\right)|\nabla u|^{p-2} \nabla u\right)+s A_{2}(x)|u|^{p s-2} u|\nabla u|^{p}=|u|^{\mu-2} u & \text { in } \Omega, \\ u=0 & \text { on } \partial \Omega .\end{cases}
$$

If $s=0$, then problem (1.4) has been widely studied (see, e.g., [16] and references therein). On the contrary, if $s>0$ we obtain the following result.

Theorem 1.1. Let $A_{1}, A_{2} \in L^{\infty}(\Omega)$ be two given functions such that

$$
A_{1}(x) \geq \alpha_{0}, \quad A_{2}(x) \geq \alpha_{0} \quad \text { for a.e. } x \in \Omega,
$$

for a constant $\alpha_{0}>0$. Assume that

$$
2<1+p<p(s+1)<\mu<p^{*}(s+1),
$$

where $p^{*}$ is the critical exponent. Then, problem (1.4) has infinitely many weak bounded solutions.

To our knowledge, there are very few results dealing with quasilinear supercritical problems. Usually, they make use of a suitable change of variables which reduces the supercritical problem to a subcritical one (see, e.g., [14). Unluckily, such an approach works only if $A(x, t, \xi)$ has a very particular form, and so, for example, it is not allowed also in the simplest case $A_{2}(x)=1$ but $A_{1}(x)$ not constant. Different arguments can be found in [4 where, by using a sequence of truncated functionals, the authors prove that problem (1.4) with, e.g., $p=2$, has at least one positive solution if (1.6) and the further condition $2(s+1)<2^{*}$ hold, which imply $N<6$ (see 4, Theorem 2.1]). Differently from [4, here we use variational methods which exploit the interaction between two different norms and we do not require this additional restriction (see also [10] where, in the same setting of Theorem 1.1, the existence of at least one positive solution of problem (1.4) is proved).

This paper is organized as follows. In Section 2 we introduce the weak Cerami-Palais-Smale condition and prove some related abstract existence and multiplicity results which generalize the Mountain Pass Theorem (see [17, Theorem 2.2]) and its symmetric version (see [17, Theorem 9.12]). In Section 3, after introducing the hypotheses for $A(x, t, \xi)$ and $G(x, t)$, we give the variational formulation of our problem and prove that $\mathcal{J}$ satisfies the weak Cerami-Palais-Smale condition. Finally, in Section 4 the main results are stated and proved.

\section{$2 \quad$ Abstract setting}

We denote $\mathbb{N}=\{1,2, \ldots\}$ and, throughout this section, we assume that:

- $\left(X,\|\cdot\|_{X}\right)$ is a Banach space with dual $\left(X^{\prime},\|\cdot\|_{X^{\prime}}\right)$,

- $\left(W,\|\cdot\|_{W}\right)$ is a Banach space such that $X \hookrightarrow W$ continuously, i.e. $X \subset W$ and a constant $\sigma_{0}>0$ exists such that

$$
\|u\|_{W} \leq \sigma_{0}\|u\|_{X} \quad \text { for all } u \in X
$$

- $J: \mathcal{D} \subset W \rightarrow \mathbb{R}$ and $J \in C^{1}(X, \mathbb{R})$ with $X \subset \mathcal{D}$,

- $K_{J}=\{u \in X: d J(u)=0\}$ is the set of the critical points of $J$ in $X$. 
Furthermore, fixing $\beta \in \mathbb{R}$, we define

- $K_{J}^{\beta}=\{u \in X: J(u)=\beta, d J(u)=0\}$ the set of the critical points of $J$ in $X$ at level $\beta$,

- $J^{\beta}=\{u \in X: J(u) \leq \beta\}$ the sublevel of $J$ with respect to $\beta$,

and, taking $r>0$, by pointing out the two different norms $\|\cdot\|_{W}$ and $\|\cdot\|_{X}$, we set

- $B_{r}^{X}=\left\{u \in X:\|u\|_{X}<r\right\}, \quad B_{r}^{W}=\left\{u \in X:\|u\|_{W}<r\right\}$,

- $\bar{B}_{r}^{X}=\left\{u \in X:\|u\|_{X} \leq r\right\}, \quad \bar{B}_{r}^{W}=\left\{u \in X:\|u\|_{W} \leq r\right\}$,

- $S_{r}^{X}=\left\{u \in X:\|u\|_{X}=r\right\}, \quad S_{r}^{W}=\left\{u \in X:\|u\|_{W}=r\right\}$.

Anyway, in order to avoid any ambiguity and simplify, when possible, the notation, from now on by $X$ we denote the space equipped with its given norm $\|\cdot\|_{X}$ while, if a different norm is involved, we write it explicitly.

For simplicity, taking $\beta \in \mathbb{R}$, we say that a sequence $\left(u_{n}\right)_{n} \subset X$ is a Cerami-Palais-Smale sequence at level $\beta$, briefly $(C P S)_{\beta}$-sequence, if

$$
\lim _{n \rightarrow+\infty} J\left(u_{n}\right)=\beta \quad \text { and } \quad \lim _{n \rightarrow+\infty}\left\|d J\left(u_{n}\right)\right\|_{X^{\prime}}\left(1+\left\|u_{n}\right\|_{X}\right)=0 .
$$

Moreover, $\beta$ is a Cerami-Palais-Smale level, briefly $(C P S)$-level, if there exists a $(C P S)_{\beta^{-}}$ sequence.

As $(C P S)_{\beta}$-sequences may exist which are unbounded in $\|\cdot\|_{X}$ but converge with respect to $\|\cdot\|_{W}$, we have to weaken the classical Cerami-Palais-Smale condition in a suitable way according to the ideas already developed in previous papers (see, e.g., [6, 7, 8]).

Definition 2.1. The functional $J$ satisfies the weak Cerami-Palais-Smale condition at level $\beta$ $(\beta \in \mathbb{R})$, briefly $(w C P S)_{\beta}$ condition, if for every $(C P S)_{\beta}$-sequence $\left(u_{n}\right)_{n}$, a point $u \in X$ exists, such that

(i) $\lim _{n \rightarrow+\infty}\left\|u_{n}-u\right\|_{W}=0 \quad$ (up to subsequences),

(ii) $J(u)=\beta, d J(u)=0$.

If $J$ satisfies the $(w C P S)_{\beta}$ condition at each level $\beta \in I, I$ real interval, we say that $J$ satisfies the $(w C P S)$ condition in $I$.

Since in [8] a Deformation Lemma has been proved if the functional $J$ satisfies a weaker version of the $(w C P S)_{\beta}$ condition, namely any $(C P S)$-level is also a critical level, in particular we can state the following result.

Lemma 2.2 (Deformation Lemma). Let $J \in C^{1}(X, \mathbb{R})$ and consider $\beta \in \mathbb{R}$ such that

- $J$ satisfies the $(w C P S)_{\beta}$ condition,

- $K_{J}^{\beta}=\emptyset$.

Then, fixing any $\bar{\varepsilon}>0$, there exist a constant $\varepsilon>0$ and a homeomorphism $\psi: X \rightarrow X$ such that $2 \varepsilon<\bar{\varepsilon}$ and 
(i) $\psi\left(J^{\beta+\varepsilon}\right) \subset J^{\beta-\varepsilon}$,

(ii) $\psi(u)=u$ for all $u \in X$ such that either $J(u) \leq \beta-\bar{\varepsilon}$ or $J(u) \geq \beta+\bar{\varepsilon}$.

Moreover, if $J$ is even on $X$, then $\psi$ can be chosen odd.

Proof. It is enough to reason as in [8, Lemma 2.3] with $\beta_{1}=\beta_{2}=\beta$ and to note that the deformation $\psi: X \rightarrow X$ is a homeomorphism.

From Lemma 2.2 we obtain the following generalization of the Mountain Pass Theorem (compare it with [8, Theorem 1.7] and the classical statement in [17, Theorem 2.2]).

Theorem 2.3. Let $J \in C^{1}(X, \mathbb{R})$ be such that $J(0)=0$ and the $(w C P S)$ condition holds in $\mathbb{R}_{+}$. Moreover, assume that there exist a continuous map $\ell: X \rightarrow \mathbb{R}$, some constants $r_{0}, \varrho_{0}>0$, and $e \in X$ such that

(i) $\quad \ell(0)=0 \quad$ and $\quad \ell(u) \geq\|u\|_{W} \quad$ for all $u \in X$;

(ii) $u \in X, \quad \ell(u)=r_{0} \quad \Longrightarrow \quad J(u) \geq \varrho_{0}$;

(iii) $\|e\|_{W}>r_{0}$ and $J(e)<\varrho_{0}$.

Then, $J$ has a Mountain Pass critical point $u_{0} \in X$ such that $J\left(u_{0}\right) \geq \varrho_{0}$.

Furthermore, with the stronger assumption that $J$ is symmetric, the following generalization of the symmetric Mountain Pass Theorem can be stated (see [8, Theorem 1.8] and compare with [17, Theorem 9.12] and [5, Theorem 2.4]).

Theorem 2.4. Let $J \in C^{1}(X, \mathbb{R})$ be an even functional such that $J(0)=0$ and the $(w C P S)$ condition holds in $\mathbb{R}_{+}$. Moreover, assume that $\varrho>0$ exists so that:

$\left(\mathcal{H}_{\varrho}\right)$ three closed subsets $V_{\varrho}, Z_{\varrho}$ and $\mathcal{M}_{\varrho}$ of $X$ and a constant $R_{\varrho}>0$ exist which satisfy the following conditions:

(i) $V_{\varrho}$ and $Z_{\varrho}$ are subspaces of $X$ such that

$$
V_{\varrho}+Z_{\varrho}=X, \quad \operatorname{codim} Z_{\varrho}<\operatorname{dim} V_{\varrho}<+\infty ;
$$

(ii) $\mathcal{M}_{\varrho}=\partial \mathcal{N}$, where $\mathcal{N} \subset X$ is a neighborhood of the origin which is symmetric and bounded with respect to $\|\cdot\|_{W}$;

(iii) $u \in \mathcal{M}_{\varrho} \cap Z_{\varrho} \quad \Longrightarrow \quad J(u) \geq \varrho$;

(iv) $u \in V_{\varrho}, \quad\|u\|_{X} \geq R_{\varrho} \quad \Longrightarrow \quad J(u) \leq 0$.

Then, if we put

$$
\beta_{\varrho}=\inf _{\gamma \in \Gamma_{\varrho}} \sup _{u \in V_{\varrho}} J(\gamma(u))
$$

with

$$
\Gamma_{\varrho}=\left\{\gamma: X \rightarrow X: \gamma \text { odd homeomeorphism, } \quad \gamma(u)=u \text { if } u \in V_{\varrho} \text { with }\|u\|_{X} \geq R_{\varrho}\right\},
$$

the functional $J$ possesses at least a pair of symmetric critical points in $X$ with corresponding critical level $\beta_{\varrho}$ which belongs to $\left[\varrho, \varrho_{1}\right]$, where $\varrho_{1} \geq \sup _{u \in V_{\varrho}} J(u)>\varrho$. 
Remark 2.5. Since in Theorem 2.4 the vector space $V_{\varrho}$ is finite dimensional, then condition $\left(\mathcal{H}_{\varrho}\right)(i v)$ implies that $\sup _{u \in V_{\varrho}} J(u)<+\infty$, furthermore it still holds if we replace $\|\cdot\|_{X}$ with $\|\cdot\|_{W}$.

If we can apply infinitely many times Theorem 2.4 then the following multiplicity abstract result can be stated.

Corollary 2.6. Let $J \in C^{1}(X, \mathbb{R})$ be an even functional such that $J(0)=0$, the $(w C P S)$ condition holds in $\mathbb{R}_{+}$and assumption $\left(\mathcal{H}_{\varrho}\right)$ holds for all $\varrho>0$.

Then, the functional $J$ possesses a sequence of critical points $\left(u_{n}\right)_{n} \subset X$ such that $J\left(u_{n}\right) \nearrow+\infty$ as $n \nearrow+\infty$.

The proof of Theorem 2.4 is obtained reasoning as in [8, Theorem 1.8] by using Lemma 2.2 and the following result.

Lemma 2.7 (Intersection Lemma). Let $V, Z$ and $\mathcal{M}$ be closed subsets of $X$ which satisfy conditions (i) and (ii) in Theorem 2.4. Fixing $R>0$ and defining

$$
\Gamma_{R}=\left\{\gamma: X \rightarrow X: \gamma \text { odd homeomeorphism, } \quad \gamma(u)=u \text { if } u \in V \text { with }\|u\|_{X} \geq R\right\},
$$

then

$$
\gamma(V) \cap \mathcal{M} \cap Z \neq \emptyset \quad \text { for all } \gamma \in \Gamma_{R} .
$$

Proof. Fixing any $\gamma \in \Gamma_{R}$, for simplicity we denote $Q=\gamma(V) \cap \mathcal{M} \cap Z$. It is enough to prove that

$$
i_{2}(Q) \geq \operatorname{dim} V-\operatorname{codim} Z \geq 1,
$$

where $i_{2}(\cdot)$ is the Krasnoselskii genus (see, e.g., [18, Section II.5]).

In order to prove (2.2), firstly let us point out that hypotheses $(i)$ and $(i i)$ imply that $Q$ is symmetric with respect to the origin but $0 \notin Q$. Moreover, $Q$ is compact in $X$. In fact, we have $V=\left(V \cap \bar{B}_{R}^{X}\right) \cup\left(V \backslash B_{R}^{X}\right)$, with $V \cap \bar{B}_{R}^{X}$ compact (as $\left.\operatorname{dim} V<+\infty\right)$ and $\gamma\left(V \backslash B_{R}^{X}\right)=V \backslash B_{R}^{X}$ (by the definition of $\left.\Gamma_{R}\right)$. Hence, $Q=\left(\gamma\left(V \cap \bar{B}_{R}^{X}\right) \cap \mathcal{M} \cap Z\right) \cup\left(\left(V \backslash B_{R}^{X}\right) \cap \mathcal{M} \cap Z\right)$ is compact because $\gamma\left(V \cap \bar{B}_{R}^{X}\right) \cap \mathcal{M} \cap Z$ is compact (as closed subset of the compact set $\gamma\left(V \cap \bar{B}_{R}^{X}\right)$ ) and $\left(V \backslash B_{R}^{X}\right) \cap \mathcal{M} \cap Z$ is compact, too, as closed and bounded in the finite dimensional space $V$ (since $\mathcal{M}$ is bounded in $\|\cdot\|_{W}$ but in $V$ the norms $\|\cdot\|_{X}$ and $\|\cdot\|_{W}$ are equivalent).

Then, by the continuity, monotonicity and subadditivity properties of the genus, an open neighborhood $U$ of $Q$ in $X$ exists such that

$$
i_{2}(Q)=i_{2}(\bar{U}) \geq i_{2}(\gamma(V) \cap \mathcal{M} \cap \bar{U}) \geq i_{2}(\gamma(V) \cap \mathcal{M})-i_{2}(\gamma(V) \cap(\mathcal{M} \backslash U)) .
$$

Now, denoting by $V^{*}$ the complement of $Z$, from hypothesis $(i)$ it follows that $V^{*} \subset V$; furthermore, it has to be $\gamma(V) \cap(\mathcal{M} \backslash U) \subset V^{*} \backslash\{0\}$, hence

$$
i_{2}(\gamma(V) \cap(\mathcal{M} \backslash U)) \leq \operatorname{dim} V^{*}=\operatorname{codim} Z .
$$

On the other hand, since $\gamma$ is an odd homeomorphism on $X$, assumption (ii) implies that the set $V \cap \gamma^{-1}(\mathcal{M})$ is the boundary of a bounded symmetric neighborhood of the origin in $V$. Then, from [18, Proposition 5.2] we have

$$
i_{2}(\gamma(V) \cap \mathcal{M})=i_{2}\left(V \cap \gamma^{-1}(\mathcal{M})\right)=\operatorname{dim} V
$$

which, together with (2.3) and (2.4), implies (2.2). 


\section{Variational setting and first properties}

From now on, let $\Omega \subset \mathbb{R}^{N}$ be an open bounded domain, $N \geq 2$, so we denote by:

- $L^{q}(\Omega)$ the Lebesgue space with norm $|u|_{q}=\left(\int_{\Omega}|u|^{q} d x\right)^{1 / q}$ if $1 \leq q<+\infty$;

- $L^{\infty}(\Omega)$ the space of Lebesgue-measurable and essentially bounded functions $u: \Omega \rightarrow \mathbb{R}$ with norm

$$
|u|_{\infty}=\underset{\Omega}{\operatorname{ess} \sup }|u|
$$

- $W_{0}^{1, p}(\Omega)$ the classical Sobolev space with norm $\|u\|_{W}=|\nabla u|_{p}$ if $1 \leq p<+\infty$;

- $|C|$ the usual Lebesgue measure of a measurable set $C$ in $\mathbb{R}^{N}$.

From now on, let $A: \Omega \times \mathbb{R} \times \mathbb{R}^{N} \rightarrow \mathbb{R}$ and $g: \Omega \times \mathbb{R} \rightarrow \mathbb{R}$ be such that, considering the notation in (1.3), the following conditions hold:

$\left(H_{0}\right) A(x, t, \xi)$ is a $C^{1}$ Carathéodory function, i.e., $A(\cdot, t, \xi): x \in \Omega \mapsto A(x, t, \xi) \in \mathbb{R}$ is measurable for all $(t, \xi) \in \mathbb{R} \times \mathbb{R}^{N}$, $A(x, \cdot, \cdot):(t, \xi) \in \mathbb{R} \times \mathbb{R}^{N} \mapsto A(x, t, \xi) \in \mathbb{R}$ is $C^{1}$ for a.e. $x \in \Omega$;

$\left(H_{1}\right)$ a real number $p>1$ and some positive continuous functions $\Phi_{i}, \phi_{i}: \mathbb{R} \rightarrow \mathbb{R}, i \in\{1,2\}$, exist such that

$$
\begin{aligned}
& \left|A_{t}(x, t, \xi)\right| \leq \Phi_{1}(t)+\phi_{1}(t)|\xi|^{p} \quad \text { a.e. in } \Omega \text {, for all }(t, \xi) \in \mathbb{R} \times \mathbb{R}^{N}, \\
& |a(x, t, \xi)| \leq \Phi_{2}(t)+\phi_{2}(t)|\xi|^{p-1} \quad \text { a.e. in } \Omega \text {, for all }(t, \xi) \in \mathbb{R} \times \mathbb{R}^{N} \text {; }
\end{aligned}
$$

$\left(G_{0}\right) g(x, t)$ is a Carathéodory function, i.e.,

$g(\cdot, t): x \in \Omega \mapsto g(x, t) \in \mathbb{R}$ is measurable for all $t \in \mathbb{R}$

$g(x, \cdot): t \in \mathbb{R} \mapsto g(x, t) \in \mathbb{R}$ is continuous for a.e. $x \in \Omega$;

$\left(G_{1}\right) a_{1}, a_{2}>0$ and $q \geq 1$ exist such that

$$
|g(x, t)| \leq a_{1}+a_{2}|t|^{q-1} \quad \text { a.e. in } \Omega \text {, for all } t \in \mathbb{R} .
$$

Remark 3.1. From $\left(G_{1}\right)$ it follows that there exist $a_{3}, a_{4}>0$ such that

$$
|G(x, t)| \leq a_{3}+a_{4}|t|^{q} \quad \text { a.e in } \Omega \text {, for all } t \in \mathbb{R} .
$$

We note that, unlike assumption $\left(G_{1}\right)$ in [7, no upper bound on $q$ is actually required.

In order to investigate the existence of weak solutions of the nonlinear problem (1.2), the notation introduced for the abstract setting at the beginning of Section 2 is referred to our problem with $W=W_{0}^{1, p}(\Omega)$ and the Banach space $\left(X,\|\cdot\|_{X}\right)$ defined as

$$
X:=W_{0}^{1, p}(\Omega) \cap L^{\infty}(\Omega), \quad\|u\|_{X}=\|u\|_{W}+|u|_{\infty}
$$

(here and in the following, $|\cdot|$ denotes the standard norm on any Euclidean space as the dimension of the considered vector is clear and no ambiguity arises). 
Moreover, from the Sobolev Embedding Theorem, for any $r \in\left[1, p^{*}\left[, p^{*}=\frac{p N}{N-p}\right.\right.$ as $N>p$, a constant $\sigma_{r}>0$ exists, such that

$$
|u|_{r} \leq \sigma_{r}\|u\|_{W} \quad \text { for all } u \in W_{0}^{1, p}(\Omega)
$$

and the embedding $W_{0}^{1, p}(\Omega) \hookrightarrow \hookrightarrow L^{r}(\Omega)$ is compact.

From the definition of $X$, we have that $X \hookrightarrow W_{0}^{1, p}(\Omega)$ and $X \hookrightarrow L^{\infty}(\Omega)$ with continuous embeddings, and (2.1) holds with $\sigma_{0}=1$. If $p>N$ then $X=W_{0}^{1, p}(\Omega)$, as $W_{0}^{1, p}(\Omega) \hookrightarrow L^{\infty}(\Omega)$; hence, classical Mountain Pass Theorems in [1] can be used.

Now, we consider the functional $\mathcal{J}: X \rightarrow \mathbb{R}$ defined as

$$
\mathcal{J}(u)=\int_{\Omega} A(x, u, \nabla u) d x-\int_{\Omega} G(x, u) d x, \quad u \in X .
$$

Taking any $u, v \in X$, by direct computations it follows that its Gâteaux differential in $u$ along the direction $v$ is

$$
\langle d \mathcal{J}(u), v\rangle=\int_{\Omega}\left(a(x, u, \nabla u) \cdot \nabla v+A_{t}(x, u, \nabla u) v\right) d x-\int_{\Omega} g(x, u) v d x .
$$

The following proposition extends [7, Proposition 3.1] in which the regularity of $\mathcal{J}$ is stated only if $G(x, t)$ has a subcritical growth.

Proposition 3.2. Let us assume that conditions $\left(H_{0}\right)-\left(H_{1}\right),\left(G_{0}\right)-\left(G_{1}\right)$ hold and two positive continuous functions $\Phi_{0}, \phi_{0}: \mathbb{R} \rightarrow \mathbb{R}$ exist such that

$$
|A(x, t, \xi)| \leq \Phi_{0}(t)+\phi_{0}(t)|\xi|^{p} \quad \text { a.e. in } \Omega, \text { for all }(t, \xi) \in \mathbb{R} \times \mathbb{R}^{N} .
$$

If $\left(u_{n}\right)_{n} \subset X, u \in X$ are such that

$$
\begin{gathered}
\left\|u_{n}-u\right\|_{W} \rightarrow 0, u_{n} \rightarrow u \text { a.e. in } \Omega \quad \text { if } n \rightarrow+\infty \\
\text { and } M>0 \text { exists so that }\left|u_{n}\right|_{\infty} \leq M \text { for all } n \in \mathbb{N} \text {, }
\end{gathered}
$$

then

$$
\mathcal{J}\left(u_{n}\right) \rightarrow \mathcal{J}(u) \quad \text { and } \quad\left\|d \mathcal{J}\left(u_{n}\right)-d \mathcal{J}(u)\right\|_{X^{\prime}} \rightarrow 0 \quad \text { if } n \rightarrow+\infty .
$$

Hence, $\mathcal{J}$ is a $C^{1}$ functional on $X$ with Fréchet differential defined as in (3.4).

Proof. As in the first part of the proof of [7, Proposition 3.1], from assumptions $\left(H_{0}\right)-\left(H_{1}\right)$ and (3.5) the functional

$$
\mathcal{A}: u \in X \mapsto \mathcal{A}(u)=\int_{\Omega} A(x, u, \nabla u) d x \in \mathbb{R}
$$

is such that $\mathcal{A}\left(u_{n}\right) \rightarrow \mathcal{A}(u)$ and $\left\|d \mathcal{A}\left(u_{n}\right)-d \mathcal{A}(u)\right\|_{X^{\prime}} \rightarrow 0$, with

$$
\langle d \mathcal{A}(u), v\rangle=\int_{\Omega} a(x, u, \nabla u) \cdot \nabla v d x+\int_{\Omega} A_{t}(x, u, \nabla u) v d x, \quad u, v \in X .
$$

On the other hand, from $\left(G_{0}\right)$ and (3.6) it follows that $G\left(x, u_{n}\right) \rightarrow G(x, u)$ and $g\left(x, u_{n}\right) \rightarrow g(x, u)$ a.e. in $\Omega$, then $\left(G_{1}\right)$, (3.1), (3.7) and Lebesgue's Dominated Convergence Theorem imply that also the functional

$$
\mathcal{G}: u \in X \mapsto \mathcal{G}(u)=\int_{\Omega} G(x, u) d x \in \mathbb{R}
$$


is such that $\mathcal{G}\left(u_{n}\right) \rightarrow \mathcal{G}(u)$ and $\left\|d \mathcal{G}\left(u_{n}\right)-d \mathcal{G}(u)\right\|_{X^{\prime}} \rightarrow 0$, with

$$
\langle d \mathcal{G}(u), v\rangle=\int_{\Omega} g(x, u) v d x \quad \text { for all } u, v \in X .
$$

Then, the conclusion follows.

In order to prove more properties of the functional $\mathcal{J}$ in (3.3), we require that some constants $\alpha_{i}>0, i \in\{1,2,3\}, \eta_{j}>0, j \in\{1,2\}$, and $s \geq 0, \mu>p, R_{0} \geq 1$, exist such that the following hypotheses are satisfied:

$\left(H_{2}\right) \quad A(x, t, \xi) \leq \eta_{1} a(x, t, \xi) \cdot \xi \quad$ a.e. in $\Omega$ if $|(t, \xi)| \geq R_{0} ;$

$\left(H_{3}\right) \quad|A(x, t, \xi)| \leq \eta_{2} \quad$ a.e. in $\Omega$ if $|(t, \xi)| \leq R_{0}$;

$\left(H_{4}\right) \quad a(x, t, \xi) \cdot \xi \geq \alpha_{1}\left(1+|t|^{p s}\right)|\xi|^{p} \quad$ a.e. in $\Omega$, for all $(t, \xi) \in \mathbb{R} \times \mathbb{R}^{N}$;

$\left(H_{5}\right) \quad a(x, t, \xi) \cdot \xi+A_{t}(x, t, \xi) t \geq \alpha_{2} a(x, t, \xi) \cdot \xi \quad$ a.e. in $\Omega$ if $|(t, \xi)| \geq R_{0}$;

$\left(H_{6}\right) \mu A(x, t, \xi)-a(x, t, \xi) \cdot \xi-A_{t}(x, t, \xi) t \geq \alpha_{3} a(x, t, \xi) \cdot \xi \quad$ a.e. in $\Omega$ if $|(t, \xi)| \geq R_{0}$;

$\left(H_{7}\right)$ for all $\xi, \xi^{*} \in \mathbb{R}^{N}, \xi \neq \xi^{*}$, it is

$$
\left[a(x, t, \xi)-a\left(x, t, \xi^{*}\right)\right] \cdot\left[\xi-\xi^{*}\right]>0 \quad \text { a.e. in } \Omega \text {, for all } t \in \mathbb{R}
$$

$\left(G_{2}\right) \quad g(x, t)$ satisfies the Ambrosetti-Rabinowitz condition, i.e.

$$
0<\mu G(x, t) \leq g(x, t) t \quad \text { for a.e. } x \in \Omega \text { if }|t| \geq R_{0} .
$$

Remark 3.3. If in $\left(H_{5}\right)$ we take $t=0$ and $|\xi| \geq R_{0}$, we deduce that $\alpha_{2} \leq 1$.

Moreover, from hypotheses $\left(H_{5}\right)$ and $\left(H_{6}\right)$ it follows that

$$
\mu A(x, t, \xi) \geq\left(\alpha_{2}+\alpha_{3}\right) a(x, t, \xi) \cdot \xi \quad \text { a.e. in } \Omega \text { if }|(t, \xi)| \geq R_{0} ;
$$

hence, if also $\left(H_{4}\right)$ holds, for a.e. $x \in \Omega$ we have that

$$
A(x, t, \xi) \geq \alpha_{1} \frac{\alpha_{2}+\alpha_{3}}{\mu}\left(1+|t|^{p s}\right)|\xi|^{p} \geq 0 \quad \text { if }|(t, \xi)| \geq R_{0} .
$$

Thus, from (3.9) and $\left(H_{3}\right)$, for a.e. $x \in \Omega$ we obtain that

$$
A(x, t, \xi) \geq \alpha_{1} \frac{\alpha_{2}+\alpha_{3}}{\mu}\left(1+|t|^{p s}\right)|\xi|^{p}-\eta_{3} \quad \text { for all }(t, \xi) \in \mathbb{R} \times \mathbb{R}^{N}
$$

for a suitable $\eta_{3}>0$.

Remark 3.4. From $\left(H_{1}\right)-\left(H_{6}\right)$, since (3.9) is verified, then

$$
|A(x, t, \xi)| \leq \eta_{1}\left(\Phi_{2}(t)+\phi_{2}(t)\right)|\xi|^{p}+\eta_{1} \Phi_{2}(t)+\eta_{2}
$$

a.e. in $\Omega$, for all $(t, \xi) \in \mathbb{R} \times \mathbb{R}^{N}$. Whence, the growth condition (3.5) holds and Proposition 3.2 applies. 
Remark 3.5. With respect to estimate (3.11), more precise growth conditions on $A(x, t, \xi)$ can be deduced. In fact, taken $|(t, \xi)| \geq R_{0}$, hypotheses $\left(H_{2}\right)$ and $\left(H_{6}\right)$ imply

$$
\mu A(x, t, \xi) \geq \frac{1+\alpha_{3}}{\eta_{1}} A(x, t, \xi)+A_{t}(x, t, \xi) t \quad \text { a.e. in } \Omega .
$$

Hence, we have

$$
\left(\mu-\frac{1+\alpha_{3}}{\eta_{1}}\right) A(x, t, \xi) \geq A_{t}(x, t, \xi) t \quad \text { a.e. in } \Omega \text { if }|(t, \xi)| \geq R_{0},
$$

where, without loss of generality, just taking $\eta_{1}$ large enough, we can always have

$$
\mu>\frac{1+\alpha_{3}}{\eta_{1}} .
$$

Thus, by means of (3.11), (3.9) and (3.12), direct calculations allow one to prove the existence of a constant $\eta_{4}>0$ so that

$$
A(x, t, \xi) \leq \eta_{4}|t|^{\mu-\frac{1+\alpha_{3}}{\eta_{1}}}|\xi|^{p} \quad \text { a.e. in } \Omega \text {, if }|t| \geq 1 \text { and }|\xi| \geq R_{0} .
$$

Whence, (3.8) and (3.13) imply

$$
a(x, t, \xi) \cdot \xi \leq \frac{\eta_{4} \mu}{\alpha_{2}+\alpha_{3}}|t|^{\mu-\frac{1+\alpha_{3}}{\eta_{1}}}|\xi|^{p} \quad \text { a.e. in } \Omega \text {, if }|t| \geq 1 \text { and }|\xi| \geq R_{0} .
$$

At last, $\left(H_{4}\right)$ and (3.14) imply that

$$
0 \leq p s \leq \mu-\frac{1+\alpha_{3}}{\eta_{1}}
$$

We note that, if

$$
0 \leq s<\frac{\mu}{p}
$$

then, without loss of generality, we can always choose $\eta_{1}$ in $\left(H_{2}\right)$ large enough so that (3.15) holds.

Remark 3.6. In the model case $A(x, t, \xi)=\frac{1}{p} \bar{A}(x, t)|\xi|^{p}$ conditions $\left(H_{2}\right)$ and $\left(H_{7}\right)$ are trivially verified, so the set of assumptions reduce to the following one:

$\left(H_{0}\right)^{\prime} \quad \bar{A}(x, t)$ is a $C^{1}$ Carathéodory function in $\Omega \times \mathbb{R}$;

$\left(H_{1}\right)^{\prime}$ two positive continuous functions $\Phi_{i}: \mathbb{R} \rightarrow \mathbb{R}, i \in\{1,2\}$, exist such that

$$
\left|\bar{A}_{t}(x, t)\right| \leq \Phi_{1}(t), \quad|\bar{A}(x, t)| \leq \Phi_{2}(t) \quad \text { a.e. in } \Omega \text {, for all } t \in \mathbb{R} ;
$$

$\left(H_{4}\right)^{\prime} \quad \bar{A}(x, t) \geq \alpha_{1}\left(1+|t|^{p s}\right) \quad$ a.e. in $\Omega, \quad$ for all $t \in \mathbb{R}$;

$\left(H_{5}\right)^{\prime} \quad \bar{A}(x, t)+\frac{1}{p} \bar{A}_{t}(x, t) t \geq \alpha_{2} \bar{A}(x, t) \quad$ a.e. in $\Omega$ if $|t| \geq R_{0}$;

$\left(H_{6}\right)^{\prime} \quad\left(\frac{\mu}{p}-1\right) \bar{A}(x, t)-\frac{1}{p} \bar{A}_{t}(x, t) t \geq \alpha_{3} \bar{A}(x, t) \quad$ a.e. in $\Omega$ if $|t| \geq R_{0}$. 
In particular, if we consider $\bar{A}(x, t)=A_{1}(x)+A_{2}(x)|t|^{p s}$ as in (1.4), the previous hypotheses hold if $A_{1}, A_{2} \in L^{\infty}(\Omega)$ are such that (1.5) is satisfied and

$$
2<1+p<p(s+1)<\mu .
$$

Remark 3.7. Conditions $\left(G_{0}\right)$ and $\left(G_{2}\right)$ imply that a function $\eta \in L^{\infty}(\Omega), \eta(x)>0$ a.e. in $\Omega$, and a constant $a_{5} \geq 0$ exist such that

$$
G(x, t) \geq \eta(x)|t|^{\mu}-a_{5} \quad \text { a.e. in } \Omega \text {, for all } t \in \mathbb{R} .
$$

Hence, if also $\left(G_{1}\right)$ holds, from (3.1), (3.16) and (3.18) it follows

$$
p s<\mu \leq q \text {. }
$$

If the assumptions in this section hold with $s=0$ in $\left(H_{4}\right)$ and $q<p^{*}$ in $\left(G_{1}\right)$, from 7 , Proposition 4.6] it follows that the functional $\mathcal{J}$ in $(3.3)$ satisfies the $(w C P S)$ condition in $\mathbb{R}$. Here, in order to extend such a result to the case $s>0$, and then considering $G(x, t)$ with a critical or supercritical growth, we need the following application of the Rellich Embedding Theorem.

Lemma 3.8. Taking $1<p<N$ and $s \geq 0$, let $\left(u_{n}\right)_{n} \subset X$ be a sequence such that

$$
\left(\int_{\Omega}\left(1+\left|u_{n}\right|^{p s}\right)\left|\nabla u_{n}\right|^{p} d x\right)_{n} \quad \text { is bounded. }
$$

Then, $u \in W_{0}^{1, p}(\Omega)$ exists such that $|u|^{s} u \in W_{0}^{1, p}(\Omega)$, too, and, up to subsequences, if $n \rightarrow+\infty$ we have

$$
\begin{aligned}
& u_{n} \rightarrow u \text { weakly in } W_{0}^{1, p}(\Omega), \\
& \left|u_{n}\right|^{s} u_{n} \rightarrow|u|^{s} u \text { weakly in } W_{0}^{1, p}(\Omega), \\
& u_{n} \rightarrow u \text { a.e. in } \Omega, \\
& u_{n} \rightarrow u \text { strongly in } L^{r}(\Omega) \text { for each } r \in\left[1, p^{*}(s+1)[.\right.
\end{aligned}
$$

Proof. Firstly, we note that

$$
\left|\nabla\left(|u|^{s} u\right)\right|^{p}=(s+1)^{p}|u|^{p s}|\nabla u|^{p} \quad \text { a.e. in } \Omega \text { for all } u \in X,
$$

then from (3.19) the sequences $\left(u_{n}\right)_{n}$ and $\left(\left|u_{n}\right|^{s} u_{n}\right)_{n}$ are bounded in $W_{0}^{1, p}(\Omega)$; hence, $u, v \in$ $W_{0}^{1, p}(\Omega)$ exist such that, up to subsequences, we have (3.20), (3.22), (3.23) with $r<p^{*}$, and also $\left|u_{n}\right|^{s} u_{n} \rightarrow v$ weakly in $W_{0}^{1, p}(\Omega)$ and $\left|u_{n}\right|^{s} u_{n} \rightarrow v$ a.e. in $\Omega$. Thus, $v=|u|^{s} u$ and (3.21) holds. At last, if $s>0$, (3.23) holds also if $p^{*} \leq r<p^{*}(s+1)$ from interpolation as $u \in L^{p^{*}(s+1)}(\Omega)$ and $\left(u_{n}\right)_{n}$ is bounded in $L^{p^{*}(s+1)}(\Omega)$.

Now, we recall a particular version of [12, Theorem II.5.1] which we will use for proving the boundedness of the weak limit of a $(C P S)$-sequence (see [7, Lemma 4.5]).

Lemma 3.9. Let $p, r$ be so that $1<p \leq r<p^{*}, p<N$ and take $v \in W_{0}^{1, p}(\Omega)$. Assume that $\bar{a}>0$ and $k_{0} \in \mathbb{N}$ exist such that the inequality

$$
\int_{\Omega_{k}^{+}}|\nabla v|^{p} d x \leq \bar{a}\left(\left|\Omega_{k}^{+}\right|+\int_{\Omega_{k}^{+}} v^{r} d x\right)
$$


holds for all $k \geq k_{0}$, with $\Omega_{k}^{+}=\{x \in \Omega: v(x)>k\}$. Then, ess $\sup v$ is bounded from above by a positive constant which can be chosen so that it depends only on $\left.\Omega \Omega\left|, N, p, r, \bar{a}, k_{0},\right| v\right|_{p^{*}}$.

Now, we are ready to prove that $\mathcal{J}$ satisfies the weak Cerami-Palais-Smale condition in $X$. If $1<p<N$, this new result extends [7. Proposition 4.6] where the exponent $q$ in $\left(G_{1}\right)$ is subcritical, i.e., $q<p^{*}$. On the contrary, here we assume the weaker condition

$$
q<p^{*}(s+1) .
$$

Hence, without loss of generality, we can always assume $q$ large enough such that

$$
p(s+1)<q<p^{*}(s+1) .
$$

Proposition 3.10. Assume that hypotheses $\left(H_{0}\right)-\left(H_{7}\right),\left(G_{0}\right)-\left(G_{2}\right)$ and (3.25) hold with $1<p<$ $N$. Then, the functional $\mathcal{J}$ satisfies the $(w C P S)$ condition in $\mathbb{R}$.

Proof. Let $\beta \in \mathbb{R}$ be fixed and consider a $(C P S)_{\beta}$-sequence $\left(u_{n}\right)_{n} \subset X$, i.e.,

$$
\mathcal{J}\left(u_{n}\right) \rightarrow \beta \quad \text { and } \quad\left\|d \mathcal{J}\left(u_{n}\right)\right\|_{X^{\prime}}\left(1+\left\|u_{n}\right\|_{X}\right) \rightarrow 0 .
$$

We divide our proof in the following steps:

1. $\left(u_{n}\right)_{n}$ is bounded in $W_{0}^{1, p}(\Omega)$, or more precisely (3.19) holds; thus from Lemma 3.8 a function $u \in W_{0}^{1, p}(\Omega)$ exists such that $|u|^{s} u \in W_{0}^{1, p}(\Omega)$ and (3.20)-(3.23) hold, up to subsequences;

2. $u \in L^{\infty}(\Omega)$;

3. if $k \geq \max \left\{|u|_{\infty}, R_{0}\right\}+1\left(R_{0} \geq 1\right.$ as in the set of hypotheses) then

$$
\mathcal{J}\left(T_{k} u_{n}\right) \rightarrow \beta \quad \text { and } \quad\left\|d \mathcal{J}\left(T_{k} u_{n}\right)\right\|_{X^{\prime}} \rightarrow 0,
$$

where $T_{k}: \mathbb{R} \rightarrow \mathbb{R}$ is the truncation function defined as

$$
T_{k} t=\left\{\begin{array}{ll}
t & \text { if }|t| \leq k \\
k \frac{t}{|t|} & \text { if }|t|>k
\end{array} ;\right.
$$

4. $\left\|T_{k} u_{n}-u\right\|_{W} \rightarrow 0$ if $n \rightarrow+\infty$, then $\left\|u_{n}-u\right\|_{W} \rightarrow 0$ if $n \rightarrow+\infty$, too;

5. $\mathcal{J}(u)=\beta$ and $d \mathcal{J}(u)=0$.

For simplicity, here and in the following we will use the notation $\left(\varepsilon_{n}\right)_{n}$ for any infinitesimal sequence depending only on $\left(u_{n}\right)_{n}$ while $d_{i}$ will denote any strictly positive constant independent of $n$.

Step 1. From (3.3), (3.4), (3.27), together with $\left(H_{1}\right),\left(H_{3}\right),\left(H_{6}\right)$, (3.1), $\left(G_{1}\right),\left(G_{2}\right)$, by reasoning as in the proof of Step 1 in [7, Proposition 4.6] and using hypothesis $\left(H_{4}\right)$ we have that

$$
\begin{aligned}
\mu \beta+\varepsilon_{n} & =\mu \mathcal{J}\left(u_{n}\right)-\left\langle d \mathcal{J}\left(u_{n}\right), u_{n}\right\rangle \geq \alpha_{3} \int_{\Omega} a\left(x, u_{n}, \nabla u_{n}\right) \cdot \nabla u_{n} d x-d_{1} \\
& \geq \alpha_{1} \alpha_{3} \int_{\Omega}\left(1+\left|u_{n}\right|^{p s}\right)\left|\nabla u_{n}\right|^{p} d x-d_{1}
\end{aligned}
$$


which implies (3.19).

Step 2. Arguing by contradiction, let us assume that

$$
\underset{\Omega}{\operatorname{ess} \sup } u=+\infty
$$

thus, taking any $k \in \mathbb{N}, k>R_{0}$ ( $R_{0} \geq 1$ as in the hypotheses), we have that

$$
\left|\Omega_{k}^{+}\right|>0 \quad \text { with } \quad \Omega_{k}^{+}=\{x \in \Omega: u(x)>k\} .
$$

Now, for any $\tilde{k}>0$ consider the new function $R_{\tilde{k}}^{+}: t \in \mathbb{R} \rightarrow R_{\tilde{k}}^{+} t \in \mathbb{R}$ such that

$$
R_{\tilde{k}}^{+} t=\left\{\begin{array}{ll}
0 & \text { if } t \leq \tilde{k} \\
t-\tilde{k} & \text { if } t>\tilde{k}
\end{array} .\right.
$$

Taking $\tilde{k}=k^{s+1}$, from (3.21) it follows that

$$
R_{k^{s+1}}^{+}\left(\left|u_{n}\right|^{s} u_{n}\right) \rightarrow R_{k^{s+1}}^{+}\left(|u|^{s} u\right) \quad \text { weakly in } W_{0}^{1, p}(\Omega) ;
$$

then, the weak lower semicontinuity of $\|\cdot\|_{W}$ implies

$$
\int_{\Omega}\left|\nabla R_{k^{s+1}}^{+}\left(|u|^{s} u\right)\right|^{p} d x \leq \liminf _{n \rightarrow+\infty} \int_{\Omega}\left|\nabla R_{k^{s+1}}^{+}\left(\left|u_{n}\right|^{s} u_{n}\right)\right|^{p} d x
$$

i.e.,

$$
\int_{\Omega_{k}^{+}}\left|\nabla\left(u^{s+1}\right)\right|^{p} d x \leq \liminf _{n \rightarrow+\infty} \int_{\Omega_{n, k}^{+}}\left|\nabla\left(u_{n}^{s+1}\right)\right|^{p} d x
$$

as $|t|^{s} t>k^{s+1} \Longleftrightarrow t>k$, with $\Omega_{n, k}^{+}=\left\{x \in \Omega: u_{n}(x)>k\right\}$.

On the other hand, from $\left\|R_{k}^{+} u_{n}\right\|_{X} \leq\left\|u_{n}\right\|_{X}$, (3.27) and (3.29) it follows that $n_{k} \in \mathbb{N}$ exists so that

$$
\left|\left\langle d \mathcal{J}\left(u_{n}\right), R_{k}^{+} u_{n}\right\rangle\right|<\left|\Omega_{k}^{+}\right| \quad \text { for all } n \geq n_{k} .
$$

From (3.4), $\left(H_{5}\right)$ with $\alpha_{2} \leq 1$ (see Remark 3.3), $\left(H_{4}\right)$, (3.24), we have that

$$
\begin{aligned}
\left\langle d \mathcal{J}\left(u_{n}\right), R_{k}^{+} u_{n}\right\rangle= & \int_{\Omega_{n, k}^{+}}\left(1-\frac{k}{u_{n}}\right)\left(a\left(x, u_{n}, \nabla u_{n}\right) \cdot \nabla u_{n}+A_{t}\left(x, u_{n}, \nabla u_{n}\right) u_{n}\right) d x \\
& \quad+\int_{\Omega_{n, k}^{+}} \frac{k}{u_{n}} a\left(x, u_{n}, \nabla u_{n}\right) \cdot \nabla u_{n} d x-\int_{\Omega} g\left(x, u_{n}\right) R_{k}^{+} u_{n} d x \\
\geq & \alpha_{2} \int_{\Omega_{n, k}^{+}} a\left(x, u_{n}, \nabla u_{n}\right) \cdot \nabla u_{n} d x-\int_{\Omega} g\left(x, u_{n}\right) R_{k}^{+} u_{n} d x \\
\geq & \alpha_{1} \alpha_{2} \int_{\Omega_{n, k}^{+}} u_{n}^{p s}\left|\nabla u_{n}\right|^{p} d x-\int_{\Omega} g\left(x, u_{n}\right) R_{k}^{+} u_{n} d x \\
= & \frac{\alpha_{1} \alpha_{2}}{(s+1)^{p}} \int_{\Omega_{n, k}^{+}}\left|\nabla\left(u_{n}^{s+1}\right)\right|^{p} d x-\int_{\Omega} g\left(x, u_{n}\right) R_{k}^{+} u_{n} d x .
\end{aligned}
$$


Thus, from (3.31) it follows that

$$
\int_{\Omega_{n, k}^{+}}\left|\nabla\left(u_{n}^{s+1}\right)\right|^{p} d x \leq \frac{(s+1)^{p}}{\alpha_{1} \alpha_{2}}\left(\left|\Omega_{k}^{+}\right|+\int_{\Omega} g\left(x, u_{n}\right) R_{k}^{+} u_{n} d x\right) .
$$

Now, from $\left(G_{1}\right),(3.23)$ and (3.25) it results

$$
\int_{\Omega} g\left(x, u_{n}\right) R_{k}^{+} u_{n} d x \rightarrow \int_{\Omega} g(x, u) R_{k}^{+} u d x
$$

hence, by passing to the lower limit, (3.30) implies

$$
\int_{\Omega_{k}^{+}}\left|\nabla\left(u^{s+1}\right)\right|^{p} d x \leq \frac{(s+1)^{p}}{\alpha_{1} \alpha_{2}}\left(\left|\Omega_{k}^{+}\right|+\int_{\Omega} g(x, u) R_{k}^{+} u d x\right) .
$$

Therefore, as in $\Omega_{k}^{+}$it is $u>1$, from $\left(G_{1}\right)$ and direct computations it follows that

$$
\int_{\Omega_{k}^{+}}\left|\nabla\left(u^{s+1}\right)\right|^{p} d x \leq d_{2}\left(\left|\Omega_{k}^{+}\right|+\int_{\Omega_{k}^{+}} u^{q} d x\right) .
$$

At last, if we set $v=|u|^{s} u$, as $v \in W_{0}^{1, p}(\Omega)$ and $\Omega_{k}^{+}=\left\{x \in \Omega: v(x)>k^{s+1}\right\}$ (in particular, $v=u^{s+1}$ in $\Omega_{k}^{+}$), from (3.32) we obtain

$$
\int_{\Omega_{k}^{+}}|\nabla v|^{p} d x \leq d_{2}\left(\left|\Omega_{k}^{+}\right|+\int_{\Omega_{k}^{+}} v^{\frac{q}{s+1}} d x\right) .
$$

Then, from (3.26) Lemma 3.9 applies and ess $\sup v<+\infty$ in contradiction to (3.28). Similar arguments apply if $\underset{\Omega}{\operatorname{ess} \sup }(-u)=+\infty$. Hence, $u \in L^{\infty}(\Omega)$.

Step 3. The proof can be obtained reasoning as in the proof of Step 3 in [7, Proposition 4.6] but using (3.23) and (3.25) instead of [7, (4.15)].

Steps 4, 5. The proofs are as in the corresponding steps of the proof of [7, Proposition 4.6].

At last, in order to prove a multiplicity result, we introduce a suitable decomposition of $X$.

If $p=2$, we deal with the Hilbert space $H_{0}^{1}(\Omega)$ so the classical choice is to consider the sequence of the eigenvalues of $-\Delta$ on $\Omega$, with homogeneous Dirichlet data, and their (bounded) eigenfunctions, so that, for each $n \geq 1$, the Banach space $X$ can be decomposed into the closed subspace spanned by the first $n$ of such eigenfunctions and the corresponding complement (for the model problem in this case, see [6]).

More in general, if $p>1$ and $p \neq 2, W_{0}^{1, p}(\Omega)$ is just a reflexive Banach space and a "canonical" decomposition is not known. Anyway, as in [7, Section 5], a sequence of positive numbers $\left(\lambda_{j}\right)_{j}$ exists such that

- $0<\lambda_{1}<\lambda_{2} \leq \cdots \leq \lambda_{j} \leq \ldots$ and $\lambda_{j} \nearrow+\infty \quad$ as $j \rightarrow+\infty$;

- for each $j \in \mathbb{N}$ a function $\varphi_{j} \in W_{0}^{1, p}(\Omega)$ exists such that $\left|\varphi_{j}\right|_{p}=1,\left\|\varphi_{j}\right\|_{W}=\lambda_{j}$ and $\varphi_{i} \neq \varphi_{j}$ if $i \neq j$; 
- $\lambda_{1}>0$ is the first eigenvalue of $-\Delta_{p}$ in $W_{0}^{1, p}(\Omega)$ such that

$$
\lambda_{1} \int_{\Omega}|w|^{p} d x \leq \int_{\Omega}|\nabla w|^{p} d x \quad \text { for all } w \in W_{0}^{1, p}(\Omega)
$$

and $\varphi_{1} \in W_{0}^{1, p}(\Omega)$ is the unique corresponding eigenfunction such that $\varphi_{1}>0,\left|\varphi_{1}\right|_{p}=1$ and $\left\|\varphi_{1}\right\|_{W}=\lambda_{1}$ (see, e.g., [13]);

- $\varphi_{j} \in L^{\infty}(\Omega)$ for each $j \in \mathbb{N}$;

- the sequence $\left(\varphi_{j}\right)_{j}$ generates the whole space $W_{0}^{1, p}(\Omega)$.

Moreover, fixing any $n \in \mathbb{N}$ and defining

$$
V_{n}=\operatorname{span}\left\{\varphi_{1}, \ldots, \varphi_{n}\right\}=\left\{v \in W_{0}^{1, p}(\Omega): \exists \beta_{1}, \ldots, \beta_{n} \in \mathbb{R} \text { s.t. } v=\sum_{i=1}^{n} \beta_{i} \varphi_{i}\right\}
$$

a closed subspace $W_{n}$ exists such that

$$
W_{0}^{1, p}(\Omega)=V_{n}+W_{n}, \quad V_{n} \cap W_{n}=\{0\},
$$

and

$$
\lambda_{n+1} \int_{\Omega}|w|^{p} d x \leq \int_{\Omega}|\nabla w|^{p} d x \quad \text { for all } w \in W_{n} .
$$

Then, $V_{n}$ is a closed subspace of $X$, too, and we have that

$$
X=V_{n}+W_{n}^{X} \quad \text { and } \quad V_{n} \cap W_{n}^{X}=\{0\}, \quad \text { with } W_{n}^{X}=W_{n} \cap L^{\infty}(\Omega),
$$

whence,

$$
\operatorname{codim} W_{n}^{X}=\operatorname{dim} V_{n}=n .
$$

\section{Existence and multiplicity results}

Finally, we can state our main theorems.

Theorem 4.1. Assume that $\left(H_{0}\right)-\left(H_{7}\right),\left(G_{0}\right)-\left(G_{2}\right)$ and (3.25) hold. If, furthermore, $\alpha_{4}>0$ exists such that

$\left(H_{8}\right) \quad A(x, t, \xi) \geq \alpha_{4}\left(1+|t|^{p s}\right)|\xi|^{p} \quad$ a.e. in $\Omega$, for all $(t, \xi) \in \mathbb{R} \times \mathbb{R}^{N} ;$

$\left(G_{3}\right) \quad \limsup _{t \rightarrow 0} \frac{g(x, t)}{|t|^{p-2} t}<p \alpha_{4} \lambda_{1}$ uniformly with respect to a.e. $x \in \Omega$, where $\lambda_{1}$ is the first eigenvalue of $-\Delta_{p}$ in $W_{0}^{1, p}(\Omega)$,

then the functional $\mathcal{J}$ defined in (3.3) possesses at least one nontrivial critical point, i.e., problem (1.2) admits at least a weak bounded nontrivial solution.

Remark 4.2. We note that the estimate in hypothesis $\left(H_{8}\right)$ follows from $\left(H_{4}\right)-\left(H_{6}\right)$ if $|(t, \xi)| \geq R_{0}$ (see inequality (3.9)). Here, we need such an estimate also for $|(t, \xi)| \leq R_{0}$. 
Theorem 4.3. Assume that $\left(H_{0}\right)-\left(H_{7}\right),\left(G_{0}\right)-\left(G_{2}\right)$ and (3.25) hold. Moreover, if $A(x, \cdot, \cdot)$ is even and $g(x, \cdot)$ is odd for a.e. $x \in \Omega$, then functional $\mathcal{J}$ in (3.3) possesses a sequence of critical points $\left(u_{n}\right)_{n} \subset X$ such that $\mathcal{J}\left(u_{n}\right) \nearrow+\infty$, i.e., problem (1.2) admits infinitely many weak bounded solutions.

We note that if $s=0$ in $\left(H_{4}\right)$ and $\left(H_{8}\right)$ or if $p \geq N$, then Theorems 4.1 and 4.3 have already been proved in 7. So, here we consider $s>0$ and $1<p<N$.

Firstly, we define

$$
\ell_{W, s}(u)=\max \left\{\|u\|_{W},\left\||u|^{s} u\right\|_{W}\right\} \quad \text { for all } u \in X .
$$

Remark 4.4. From (3.2) it follows that the map $u \mapsto|||u|^{s} u \|_{W}$ is well-defined and continuous in $\left(X,\|\cdot\|_{X}\right)$; thus, also $\ell_{W, s}: X \rightarrow \mathbb{R}$ is continuous with respect to $\|\cdot\|_{X}$.

From now on, assume that $\left(H_{0}\right)-\left(H_{7}\right),\left(G_{0}\right)-\left(G_{2}\right)$ and (3.25) hold.

In order to prove that $\mathcal{J}$ satisfies some suitable geometric conditions, we need the following lemmas.

Proposition 4.5. Fixing any $\varrho \in \mathbb{R}$ there exist $n \in \mathbb{N}, n=n(\varrho)$, and $r_{n}>0$ such that

$$
u \in W_{n}^{X}, \quad \ell_{W, s}(u)=r_{n} \quad \Longrightarrow \quad \mathcal{J}(u) \geq \varrho,
$$

where the subspace $W_{n}^{X}$ is as in (3.35).

Proof. Firstly, taking any $u \in X$ we note that (3.1) and (3.10) imply

$$
\mathcal{J}(u) \geq \alpha_{1} \frac{\alpha_{2}+\alpha_{3}}{\mu} \int_{\Omega}\left(1+|u|^{p s}\right)|\nabla u|^{p} d x-a_{4} \int_{\Omega}|u|^{q} d x-\left(\eta_{3}+a_{3}\right)|\Omega|,
$$

while from (3.24) and (4.1) it follows that

$$
\int_{\Omega}\left(1+|u|^{p s}\right)|\nabla u|^{p} d x \geq \frac{1}{(s+1)^{p}}\left[\ell_{W, s}(u)\right]^{p} .
$$

On the other hand, from (3.26), a constant $r>0$ exists such that $\frac{r}{p}+\frac{q-r}{p^{*}(s+1)}=1$, so, classical interpolation arguments apply and we have

$$
|u|_{q}^{q} \leq|u|_{p^{*}(s+1)}^{q-r}|u|_{p}^{r},
$$

where, by the Sobolev Embedding Theorem and (4.1), one has

$$
|u|_{p^{*}(s+1)}^{q-r}=\left\|\left.\left.u\right|^{s} u\right|_{p^{*}} ^{\frac{q-r}{s+1}} \leq c_{*}\right\||u|^{s} u \|_{W}^{\frac{q-r}{s+1}} \leq c_{*}\left[\ell_{W, s}(u)\right]^{\frac{q-r}{s+1}}
$$

for a suitable constant $c^{*}>0$.

Now, fixing any $n \in \mathbb{N}$, from (3.34), (4.1), (4.5) and (4.6) it follows that

$$
|u|_{q}^{q} \leq c_{*} \lambda_{n+1}^{-\frac{r}{p}}\|u\|_{W}^{r}\left[\ell_{W, s}(u)\right]^{\frac{q-r}{s+1}} \leq c_{*} \lambda_{n+1}^{-\frac{r}{p}}\left[\ell_{W, s}(u)\right]^{r+\frac{q-r}{s+1}} \quad \text { for all } u \in W_{n}^{X},
$$

where from (3.26) we have

$$
r+\frac{q-r}{s+1}=\frac{q+r s}{s+1}>p .
$$


Hence, (4.3), (4.4) and (4.7) imply that

$$
\begin{aligned}
\mathcal{J}(u) & \geq b_{1}\left[\ell_{W, s}(u)\right]^{p}-b_{2} \lambda_{n+1}^{-\frac{r}{p}}\left[\ell_{W, s}(u)\right]^{\frac{q+r s}{s+1}}-b_{3} \\
& =b_{1}\left[\ell_{W, s}(u)\right]^{p}\left(1-\frac{b_{2}}{b_{1}} \lambda_{n+1}^{-\frac{r}{p}}\left[\ell_{W, s}(u)\right]^{\frac{q+r s}{s+1}-p}\right)-b_{3} \quad \text { for all } u \in W_{n}^{X},
\end{aligned}
$$

for suitable positive constants $b_{1}, b_{2}, b_{3}$ independent of $n$.

Finally, we choose $r_{n}>0$ so that

$$
1-\frac{b_{2}}{b_{1}} \lambda_{n+1}^{-\frac{r}{p}} r_{n}^{\frac{q+r s}{s+1}-p}=\frac{1}{2}
$$

i.e.,

$$
r_{n}=\left(\frac{b_{1}}{2 b_{2}} \lambda_{n+1}^{\frac{r}{p}}\right)^{\frac{s+1}{q-p(s+1)+r s}} .
$$

Thus, as $\lambda_{n} \nearrow+\infty,(3.26)$ and (4.8) imply that $r_{n} \nearrow+\infty$, then from the estimate

$$
\mathcal{J}(u) \geq \frac{b_{1}}{2} r_{n}^{p}-b_{3} \quad \text { for all } u \in W_{n}^{X} \text { with } \ell_{W, s}(u)=r_{n},
$$

the thesis follows.

At last, as in [7, Proposition 6.6], the following statement holds.

Proposition 4.6. For any finite dimensional subspace $V$ of $X$, there exists $R>0$ such that

$$
\mathcal{J}(u) \leq 0 \quad \text { for all } u \in V \text { with }\|u\|_{X} \geq R .
$$

Hence, $\mathcal{J}$ is bounded from above in $V$.

Proof of Theorem 4.1. From $\left(G_{3}\right)$, we can take $\bar{\lambda} \in \mathbb{R}$ so that

$$
\limsup _{t \rightarrow 0} \frac{g(x, t)}{|t|^{p-2} t}<\bar{\lambda}<p \alpha_{4} \lambda_{1} .
$$

Then, from $\left(G_{1}\right)$, (4.9) and standard computations, a suitable constant $b_{1}>0$ exists such that

$$
G(x, t) \leq \frac{\bar{\lambda}}{p}|t|^{p}+b_{1}|t|^{q} \quad \text { a.e. in } \Omega \text {, for all } t \in \mathbb{R} ;
$$

hence, from $\left(H_{8}\right)$, (3.24) and (3.33), we obtain

$$
\mathcal{J}(u) \geq\left(\alpha_{4}-\frac{\bar{\lambda}}{p \lambda_{1}}\right)\|u\|_{W}^{p}+\frac{\alpha_{4}}{(s+1)^{p}}\left\||u|^{s} u\right\|_{W}^{p}-b_{1}|u|_{q}^{q} \quad \text { for all } u \in X,
$$

with $\alpha_{4}-\frac{\bar{\lambda}}{p \lambda_{1}}>0$. Now, since (3.25) holds, by Sobolev Embedding Theorem and (4.1), we have

$$
\int_{\Omega}|u|^{q} d x=\left.\left.\int_{\Omega}|| u\right|^{s} u\right|^{\frac{q}{s+1}} d x \leq b_{2}\left\||u|^{s} u\right\|_{W}^{\frac{q}{s+1}} \leq b_{2}\left[\ell_{W, s}(u)\right]^{\frac{q}{s+1}}
$$


for some $b_{2}>0$. Thus, from the previous estimates it follows that there exist $b_{3}, b_{4}>0$ such that

$$
\mathcal{J}(u) \geq b_{3}\left[\ell_{W, s}(u)\right]^{p}-b_{4}\left[\ell_{W, s}(u)\right]^{\frac{q}{s+1}} \quad \text { for all } u \in X .
$$

Whence, from (3.26) some strictly positive constants $r_{0}, \varrho_{0}>0$ can be chosen so that $\mathcal{J}(u) \geq \varrho_{0}$ if $\ell_{W, s}(u)=r_{0}$.

On the other hand, taking any $v^{*} \in X \backslash\{0\}$, by Proposition 4.6 with $V=\operatorname{span}\left\{v^{*}\right\}$ and the equivalence of $\|\cdot\|_{X}$ and $\|\cdot\|_{W}$ in $V$, an element $e \in V$ exists such that $\|e\|_{W}>r_{0}$ and $\mathcal{J}(e) \leq 0$. Whence, as without loss of generality we can assume $\int_{\Omega} A(x, 0,0) d x=0$, it is $\mathcal{J}(0)=0$, so Proposition 3.10 and Theorem 2.3 imply that $\mathcal{J}$ has at least a nontrivial critical point.

Proof of Theorem 4.3. For simplicity, if $r>0$ we set

$$
\mathcal{M}_{r}=\left\{u \in X: \ell_{W, s}(u)=r\right\} .
$$

We note that $\mathcal{M}_{r}$ is the boundary of a neighborhood of the origin which is symmetric and bounded with respect to $\|\cdot\|_{W}$.

Then, fixing any $\varrho>0$, from Proposition 4.5 an integer $n \in \mathbb{N}$ and a constant $r_{n}>0$ exist such that (4.2) holds, i.e.

$$
u \in \mathcal{M}_{r_{n}} \cap W_{n}^{X} \Longrightarrow \mathcal{J}(u) \geq \varrho .
$$

Now, taking any $m>n$, from (3.36) the $m$-dimensional space $V_{m}$ is such that $\operatorname{codim} W_{n}^{X}<\operatorname{dim} V_{m}$; thus, Proposition 4.6 and the previous remarks imply that assumption $\left(\mathcal{H}_{\varrho}\right)$ in Theorem 2.4 holds. At last, without loss of generality we can assume $\int_{\Omega} A(x, 0,0) d x=0$, then $\mathcal{J}(0)=0$ and for the arbitrariness of $\varrho>0$ and Proposition 3.10 we have that Corollary 2.6 applies.

Proof of Theorem 1.1. The proof follows from Theorem 4.3 and Remark 1.6 with $g(x, t)=|t|^{\mu-2} t$ and so $q=\mu$.

\section{References}

[1] A. Ambrosetti and P.H. Rabinowitz, Dual variational methods in critical point theory and applications, J. Funct. Anal. 14 (1973), 349-381.

[2] D. Arcoya and L. Boccardo, Critical points for multiple integrals of the calculus of variations, Arch. Rational Mech. Anal. 134 (1996), 249-274.

[3] D. Arcoya and L. Boccardo, Some remarks on critical point theory for nondifferentiable functionals, NoDEA Nonlinear Differential Equations Appl. 6 (1999), 79-100.

[4] D. Arcoya, L. Boccardo and L. Orsina, Critical points for functionals with quasilinear singular Euler-Lagrange equations, Calc. Var. Partial Differential Equations 47 (2013), 159-180.

[5] P. Bartolo, V. Benci and D. Fortunato, Abstract critical point theorems and applications to some nonlinear problems with "strong" resonance at infinity, Nonlinear Anal. 7 (1983), 981-1012. 
[6] A.M. Candela and G. Palmieri, Multiple solutions of some nonlinear variational problems, Adv. Nonlinear Stud. 6 (2006), 269-286.

[7] A.M. Candela and G. Palmieri, Infinitely many solutions of some nonlinear variational equations, Calc. Var. Partial Differential Equations 34 (2009), 495-530.

[8] A.M. Candela and G. Palmieri, Some abstract critical point theorems and applications. In: Dynamical Systems, Differential Equations and Applications (X. Hou, X. Lu, A. Miranville, J. Su \& J. Zhu Eds), Discrete Contin. Dynam. Syst. Suppl. 2009 (2009), 133-142.

[9] A.M. Candela and G. Palmieri, Multiplicity results for some nonlinear elliptic problems with asymptotically $p$-linear terms, Calc. Var. Partial Differential Equations 56:72 (2017).

[10] A.M. Candela and A. Salvatore, Positive solutions for some generalized $p$-Laplacian type problems, Discrete Contin. Dyn. Syst. Ser. S (to appear) .

[11] A. Canino, Multiplicity of solutions for quasilinear elliptic equations, Topol. Methods Nonlinear Anal. 6 (1995), 357-370.

[12] O.A. Ladyzhenskaya and N.N. Ural'tseva, Linear and Quasilinear Elliptic Equations, Academic Press, New York, 1968.

[13] P. Lindqvist, On the equation $\operatorname{div}\left(|\nabla u|^{p-2} \nabla u\right)+\lambda|u|^{p-2} u=0$, Proc. Amer. Math. Soc. 109 (1990), 157-164.

[14] J.Q. Liu, Y.Q. Wang and Z.Q. Wang, Soliton solutions for quasilinear Schrödinger equations, II, J. Differential Equations 187 (2003), 473-493.

[15] B. Pellacci and M. Squassina, Unbounded critical points for a class of lower semicontinuous functionals, J. Differential Equations 201 (2004), 25-62.

[16] K. Perera, R.P. Agarwal and D. O'Regan, Morse Theoretic Aspects of p-Laplacian Type Operators, Math. Surveys Monogr. 161, Amer. Math. Soc., Providence RI, 2010.

[17] P.H. Rabinowitz, Minimax Methods in Critical Point Theory with Applications to Differential Equations, CBMS Regional Conf. Ser. in Math. 65, Amer. Math. Soc., Providence, 1986.

[18] M. Struwe, Variational Methods. Applications to Nonlinear Partial Differential Equations and Hamiltonian Systems, 4rd Edition, Ergeb. Math. Grenzgeb. (4) 34, Springer-Verlag, Berlin, 2008. 